\title{
A VIEW ON EXPERIENCE AND COMMUNICATION PERSPECTIVES IN LUNG CANCER PATIENTS IN CROATIA
}

\author{
Sandra Karabatić ${ }^{1}$, Andreja Šajnić ${ }^{1}$, Vanesa Benković², Marko Jakopović ${ }^{1,3}$, Miroslav Samaržija ${ }^{1,3}$ \\ ${ }^{1}$ Dept. for Respiratory Diseases Jordanovac, University Hospital Center Zagreb, Croatia \\ ${ }^{2}$ Croatian Society for Pharmacoeconomics and Health Economics, Drage Stipca 10, 10090 Zagreb, Croatia \\ ${ }^{3}$ University of Zagreb School of Medicine, Zagreb, Croatia \\ Rad je primljen 25.06.2021. Rad je recenziran 16.07.2021. Rad je prihvaćen 25.07.2021.
}

\begin{abstract}
Introduction: Well-balanced communication between healthcare professionals and oncology patients is the first step toward goals of caring for patients.

Aims: In constant aim to improve patient outcomes, we aspired to uncover shortcomings in communication between healthcare professionals and patients suffering from lung cancer. An online survey of a non-probabilistic sample of cancer patients from twelve countries of Central and Eastern Europe designed and implemented by the Croatian Coalition of Associations in Healthcare.

Methods: 472 lung cancer subjects were selected from the sample of results from 2,460 Croatian participants in the study from Oct. 2018. to Feb. 2019. Participants' sociodemographic and clinical characteristics, experience and communication with healthcare professionals, key areas of inefficiency, psychological and social support were assessed. The analysis included descriptive statistics and chi square to assess demographic differences.

Results: The study revealed four main results: (i) only a minority of the participants were satisfied with the communication process, (ii) majority were not told that they could bring family member during delivering bad news, (iii) there was a clear unmet need to have a discussion on the impact of cancer on day to day activities (iv) participants' preferences indicate that the greatest improvement would be to perform all services at one place.

Conclusions: This study revealed that one of the leading problems in oncology care according to patients is the lack of education and effective communication. Based on the obtained data, we conclude that there is a large space for improvement in patient experience and communication.

Key words: communication, lung cancer, patient's experience

Corresponding author

Sandra Karabatić, MScN, RN, PhD student

Dept. for Respiratory Diseases Jordanovac,

University Hospital Center, Zagreb, Croatia

Phone +385911830493

udruga.jedra@gmail.com
\end{abstract}




\section{INTRODUCTION}

The global cancer mortality rate has been steadily declining from 1991. to 2017., resulting in an overall decline of $29 \%$, resulting in an estimated 2.9 million fewer cancer deaths. Long-term reductions in mortality rates are for the four leading cancers (lung, colorectal, breast, prostate). Declines accelerated for lung cancer, yet lung cancer still is expected to be most diagnosed in men and women and it caused more deaths in 2017. than breast, prostate, colorectal, and brain cancers combined. The 5-year survival rate for lung cancer still remains in the lowest range (19\%). Low lung cancer survival rates reflect the large proportion of patients (57\%) diagnosed with metastatic disease, for which the 5 -year relative survival rate is $5 \%$. However, the 5 -year survival rate for localized stage disease is $57 \%(1)$.

Aside from the obvious clinical symptoms, patients with lung cancer can suffer significant psychological impacts (2), experiencing a greater level of distress than that associated with other prevalent cancers, possibly because of the poor prognosis of lung cancer (3). Wellbalanced communication between healthcare professionals (HCPs) and oncology patients is the first step toward goals of caring for patients and increasing daily efficiency as well as overcoming obstacles in upcoming treatment and care. Effective communication is linked to patient experience and it is the key component which impacts patient satisfaction, safety, pain control, adherence and clinical health outcomes (4). During the past it was assumed that well-balanced communication was an inborn quality of HCPs and that there is no need for a formal education; now formal curriculum for HCPs is focused more on quality, safety, and patient experiences of care (5). Ineffective communication still often goes undetected in many healthcare settings, causing serious effects on health and safety of patients. Data suggests that patients want more involvement in their care, there is a clear need for active involvement of patients in the decision-making process (6). Transition from a paternalistic model to a model based on informed shared decision-making (SDM) is enabling implementation of integrative health care delivery in the ethical way, preserving patient autonomy, considering patient values and preferences and directing toward improved health outcomes (7). SDM has been proven to have beneficial effects on patient outcomes such as patient satisfaction, well-being, and quality of life (8).

Effective communication skills interfere with the creation of patient-centred care, and there is a clear need for HCPs communication skills training programs with focus on psychosocial issues, especially for HCPs which works with oncological patients (9). Both American and European clinical guidelines (ASCO and ESMO) recommend communication skills training for oncologists and presents best practices for core communication skills when clinicians are communicating with patients and their loved ones about goals of care, prognosis, treatment options, and end-of-life care $(10,11)$.

For every skill to be trained there is a need to find out what are the needs and perspectives from the patients and the lack of the specific skill in the healthcare system. In the period from October 2018. to February 2019. Croatian Coalition of Associations in Healthcare, designed and implemented an international online survey on patient experience during oncology treatment from twelve countries of Central and Eastern Europe (CEE). An interactive internet-based tool was used to gather free-text and multiple-choice responses illustrating the views and experiences of cancer patients. The study aimed to identify the needs and perspectives of oncological patients during the cancer treatment. In this paper we have focused on a subpopulation of Croatian lung cancer patients. Aiming for the best possible patient outcomes, we aspired to uncover shortcomings in communication between $\mathrm{HCPs}$ and patients suffering from lung cancer in order to provide proposals for improvement in communication and lay ground for improving patient experience.

\section{METHODS}

Study design, data collection and analysis, participants From the initial questionnaire composed of 69 questions, developed by the Croatian Coalition of Associations in Healthcare, we have selected questions that we grouped to reflect dimensions of patient experience, communication, self-management and identification of area of improvements. The dimensions of patient experience and communication include experiences while receiving cancer diagnosis, perceptions and the perceived impact of cancer on their life including perceived support from HCPs, family and colleagues from work, including thoughts and feelings regarding dying and death. Series of questions related to self-management included the care and impact of cancer on their everyday life. Selected questions included sociodemographic data (sex, age, place of residence, working status), data on diagnosis, patient experience (How did you feel when you got your diagnosis? Did the staff discuss your diagnosis as if you were not there? Were you told to bring a family member or a friend to a doctor appointment? etc.) and communication (How many 
conversations did you have since the start of your therapy? Was the plan of treatment explained to you? Did you get the information on impact on your daily activities? etc.). Also, open ended questions allowed for suggestions on inefficiency of communication and recommendations for improvement of patient care. Data gathered were in the form of both open ended free text responses to enable eliciting key experiences, thoughts and feelings as well as fixed choices from a selection of on-screen options. Apart from descriptive statistics, more detailed insight was enabled using chi square test where all of the differences of given values were analysed on the level of 5\% significance.

The initial online survey recruited 16,458 participants from twelve countries of CEE via national patient (oncology) associations. A total of 2,460 Croatian cancer patients participated in the online survey, whereas in this paper, we used the data based on the responses of 472 subjects with lung cancer. Recruitment of participants was provided by patient associations who enrolled participants over the phone or by providing links via official web pages and national patient oncology associations' social media (Facebook). Inclusion criteria for patients included confirmed cancer diagnosis and the ability to access the internet.

\section{RESULTS}

Survey data was obtained from 472 espondents from Croatia. Table 1. provides an overview of demographic of study population, out of which there were $44 \%$ males and $56 \%$ females with average age of $61.55 \pm 7.18$ years. The youngest respondent was 43 years and the oldest one 82 years. Out of 472 respondents $42 \%$ respondents were treated in only one city and most of them were treated in the capitol Zagreb (32\%).

Table 1. Respondents demographics $(\mathrm{N}=472)$

$\begin{array}{lcc}\text { Demographics } & \mathrm{N} & \% \\ \text { Total respondents } & 472 & 100.0 \\ \text { Male } & 206 & 44 \\ \text { Female } & 266 & 56 \\ \text { Employment status } & & \\ \text { Fully employed } & 121 & 25.6 \\ \text { Part time employed } & 6 & 1.3 \\ \text { Unemployed } & 72 & 15.3 \\ \text { Sick leave } & 89 & 18.9 \\ \text { Retired } & 165 & 35.0 \\ \text { Other } & 19 & 4.0 \\ \text { Total } & 472 & 100.0\end{array}$

\section{Patient experience}

Sixty-five percent $(n=305 / 472)$ of respondents had claimed that they had not been told they could bring a family member or a friend with them, and only part of them (24\%) were told to bring. Further, over half of the respondents (54\%) experienced that the way they were told that they had cancer was done sensitively and $25 \%$ experienced that it should have done a lot more sensitively. In addition, there were respondents saying that it could have been done a bit more sensitively (21\%). Experience of being disconnected from decision making was expressed in majority of respondents (65\%) as it is presented in Table 2. 
Table 2. Experience of being disconnected

\begin{tabular}{lcc}
\hline Did groups of doctors and nurses talk in front of you as if you weren't there? & $\mathrm{N}$ & $\%$ \\
\hline Yes, often & 28 & 5.9 \\
Yes, sometimes & 114 & 24.2 \\
No & 308 & 65.3 \\
Don't know / can' remember & 22 & 4.7 \\
Total & 472 & 100.0 \\
\hline
\end{tabular}

There was a statistically significant difference $(x(3)=8.857, p=.031)$ among men and women, where women experienced more disconnect than men.

\section{Communication}

When having communicated the treatment options, majority of patients claimed that they had had their treatment options explained to some extent (40\%), completely explained (29\%) and without any explanation at all (18\%). For this variable, we have found a statistically significant difference in female respondents $(x$ $(4)=15.208, p=.004)$ and part of it might be attributed to the assumption that these respondents were more willing to communicate per se.

In Croatia communication around the cancer treatment plan should be a regular practice which was claimed by $49 \%$ of patients, whereas $38 \%$ of patients were not communicated the cancer plan. As part of the patients said they did not understand what the cancer treatment plan was at all (13\%) we could not have concluded whether it was communicated to them or not. A very important part of the treatment plan is communication about the side effects which should be provided. The majority of patients remember they were explained in a way they could understand $(27 \%$ were sure of it and $41 \%$ claimed that it was explained to some extent and still part of the patients claimed that no side effects were explained at all (28\%).

\section{Communicating about impact of cancer on day to day activities}

Series of questions related to self-management included the care and impact of cancer on their everyday life. There was a clear unmet need to have a discussion on the impact of cancer on day to day activities (see Table 3.).

Table 3. Communicating about impact of cancer on day to day activities

\begin{tabular}{lrr}
\hline Did hospital staff discuss with you or give you information about the impact cancer could have on your day to day & N & $\%$ \\
activities & 131 & 27.8 \\
\hline Yes & 257 & 54.4 \\
No, but I would have liked a discussion or information & 76 & 16.1 \\
It was not necessary / relevant to me & 8 & 1.7 \\
Don't know / can't remember & 472 & 100.0 \\
Total
\end{tabular}




\section{Nonclinical care and support given during treatment}

Part of the nonclinical care and support given during treatment was covered asking the respondents whether they were worried about their condition or treatment after discharge and most of them (64\%) were not given any contact unlike $32 \%$ who stated they were given the contact if worried. When asked whether they found someone among the hospital staff to talk to about their worries and fears, the respondents' answers were split in half; half of them did not find (51\%) and the other part did find (48\%). From this question it is not clear how these results correlate to the actual fact that they did search for someone to talk to. As for the information on the support from health or social services (district nurses, home helps, psychological support) again over half of them (57\%) responded that there was not enough care or support and only small part of respondents (6\%) had been given the care or at least to some extent (17\%) (see Table 4.).

Table 4. Nonclinical care and support given during treatment

\begin{tabular}{lrr}
\hline During your cancer treatment, and once your cancer treatment finished, were you given enough care and support from & N & $\%$ \\
health or social services (for example, district nurses, home helps, psychological support)? & 28 & 5.9 \\
\hline Yes, definitely & 80 & 16.9 \\
Yes, to some extent & 271 & 57.4 \\
No & 83 & 17.6 \\
Idid not need help from health or social services & 462 & 97.9 \\
Total &
\end{tabular}

\section{Patients' views on oncology treatment and health care}

To be able to identify key points where the care and treatment may improve, the respondents were asked where they had felt the highest rate of inefficiency. They were offered 10 options and they could select all that applied to them.
Largest share of respondents felt that highest inefficiency was in communication among different HCPs involved in their cancer care $(46.2 \%)$. As a confirmation that communication is the issue that should be pinpointed, the smallest share of respondents felt there was an issue with their treatment (see Table 5.). 
Table 5. Inefficiencies in care and treatment

\begin{tabular}{lrc}
\hline During the whole of your cancer care and treatment, where do you feel there was most inefficiency? & $\mathrm{N}$ & $\%$ \\
\hline My initial cancer diagnosis & 14 & 30.5 \\
& 4 & 17.6 \\
Getting the right treatment for my cancer & 83 & 36.2 \\
Dealing with ongoing side effects & 171 \\
& & 34.1 \\
Dealing with the financial implications & 16 & 1 \\
& 19 & 40.7 \\
Dealing with the psychological impacts & 2 & 22.5 \\
& 10 & \\
Access to patient support groups & 6 & 26.9 \\
& & \\
The opportunity to take part in clinical trials & 12 & 21.8 \\
Supportive therapy & 10 & \\
& & 32.7 \\
Palliative care & 30 & 46.2 \\
Communications between different people involved in your cancer care & 21 & 8 \\
\end{tabular}

When asked to express how the decision making related to their diagnosis could be improved there was an option to select three out of four options. Most of the respondents felt that the greatest improvement would be to perform all services at one place well as to have a multidisciplinary team. In this question it seems that patient's value more the organization and management of care rather than the communication of their diagnosis (see Table 6.).

Table 6. Improvement proposals

\begin{tabular}{lcc}
\hline & $\mathrm{N}$ & $\%$ \\
By doing everything in one healthcare institution & 288 & 61.0 \\
By multidisciplinary team coordinated by one person & 267 & 56.6 \\
Shorter period of waiting for the results & 222 & 47.0 \\
The way my diagnosis is communicated & 85 & 18.0 \\
\hline
\end{tabular}




\section{Discussion}

Patients experience in delivering bad news reflects how will they adjust to diagnosis and treatment. An inappropriate way of communicating with the patient can have a huge impact on the way they perceive their disease, it can also influence whether they quit or continue the medical treatment (12). Patients differ in what information they want and how they want to receive it. Many oncological patients reported differences in receiving information between requested and realization. In one study $42.3 \%$ of the patients stated that it is not possible to make important decisions during the first bad-news discussion and 50.2\% wished to have a second talk, preferably accompanied by a relative or loved one, there was lower patient satisfaction in the way bad news were communicated, only $46.1 \%$ were satisfied with disclosure process of their cancer diagnosis. Only 46 percent of patients were satisfied with the communication regarding addressing emotions and in only 41 percent of cases were given a clear explanation of the diagnosis from their provider (13). In our study, $65 \%$ of respondents reported they didn't know that they could bring a relative or loved one during the first bad-news discussion. Experience of being disconnected from decision making was expressed in majority of respondents $(65 \%)$, there was a statistically significant difference among men and women, where women experienced more disconnect than men.

Shared decision-making (SDM )is a strategy to facilitate patient-centered care and is increasingly important in oncology, where patients are faced with complicated treatment decisions that require them to weigh efficacy and safety, quality of life, and cost (14). In one study, patients with early stage non-small cell lung cancer reported a lack of knowledge about the advantages and disadvantages of treatment options, however, $74 \%$ still felt they were sufficiently involved in decision-making (15). Majority of our patients had their treatment options explained to some extent (40\%), completely explained (29\%) and without any explanation at all (18\%); (38\%) of patients were without any explanation at all about their cancer treatment plan and $(28 \%)$ did not have any of therapy side effects explained at all. With advances in treatments among patients with lung cancer, it is increasingly important to understand patients' values and preferences to facilitate shared decision making (16). In one a systematic literature review of patient perspective of SDM in oncology care, three consistent themes of barriers to SDM emerged across the studies, including uncertainty or lack of consensus in the treatment decision, patient concern regarding adverse effects, and poor physician communication (14). Ineffective communication can increase patients' level of anxiety and displeasure with care; lack of therapy compliance and poorer quality of life (17). In a study of 1,193 patients with metastatic lung and colorectal cancer, 69 percent of those with lung cancer did not report understanding that chemotherapy was not at all likely to cure their cancer (18). Communication between healthcare professional and patients' needs to be focused on providing information's about prognosis prior to commending to treatment, check patients understanding, provide an opportunity to ask questions and explaining medical terms. Self-management (SM) defined as a person's ability to manage the symptoms and the consequences of living with a chronic condition, including treatment, physical, social and lifestyle changes. SM as a model of cancer care that involves providers forming partnerships with patients and families. These partnerships enable and empower patients and families to achieve their own goals of care at all phases along the cancer-care continuum (19).The role of nurses in delivering SM interventions with positive outcomes has been confirmed in one study were standard nursing intervention protocol has been delivered by advanced practice oncology nurses on older post-surgical cancer patients' physical, functional, emotional, and psychological recovery in the home setting. SM interventions significantly reduced uncertainty in the intervention group; the intervention sub-group that received additional psychological support showed significant improvement in mental and physical QOL, less uncertainty, and less symptom distress (20). Oncology patients report negative feelings and experiences associated with certain clinician behaviours such as giving inadequate information, rushing the patient for a treatment decision, and not addressing patients' feelings about the news (21). In our study, most of the patients didn't receive adequate information were and whom to address when they feel worried or in fear; (64\%) were not given any information to whom to address and only $32 \%$ stated they receive adequate information. Only (51\%) respondents found someone among the hospital staff to talk to about their worries and fears. Patient satisfaction with provided services was low, only (57\%) responded stated that there was not enough care or support from HCPs (see Table 4.). There was a clear unmet need to have a discussion on the impact of cancer on daily lives (54.4\% had no discussion). Studies shown when clinicians express concern about patient feelings at the time delivering bad news, perception about their disease, problems and impact of illness on daily lives there were significantly less level of anxiety, greater satisfaction and 
treatment compliance (22).

Oncology patients want emotional support from physicians (23), their caregivers involved in their care (24), accessible information sharing, and to participate in the decision-making during their cancer treatment (9). Patient well-being is higher when teamwork is improved between nurses and physicians (6). Healthcare professional empathic communication is associated with higher rates of patients' satisfaction and lower levels of psychological distress (25). Largest share of respondents in our study felt the highest inefficiency was in communication among HCPs involved in their cancer care $(46.2 \%)$, dealing with the psychological impacts (40.7\%) and with ongoing side effects (36.2\%). Patients propose improvements by doing everything in one healthcare institution (61\%), multidisciplinary team coordinated by one person (56.6\%), shorter period of waiting for the results (47\%) and the way diagnosis is communicated with them (18\%). It seems that patient's value more the organization and management of care rather than the communication of their diagnosis (see Table 6.).

\section{CONCLUSIONS}

In conclusion, this study adds to the current understanding on the importance of patient experience and effective bidirectional communication. Results from this survey on lung cancer patient experience that there is a large room for improvement in this area. Only a minority of the asked cancer patients were satisfied with the communication process and the way the treatment and side effects were communicated to them. Overall, this study adds to current understanding, to build therapeutic alliance between healthcare professionals and patients it is necessary to achieve effective communication. Oncology patients need communication that allows them to feel guided, build trust, and sustain hope. Effective communication skills in healthcare sector are essential for patient experience and essential for the overall quality and safety care.

\section{REFERENCES}

1. Siegel RL, Miller KD, Jemal A. Cancer statistics, 2020. CA Cancer J Clin. 2020;70(1):7-30.

2. Buchanan D, Milroy R, Baker L, Thompson AM, Levack PA. Perceptions of anxiety in lung cancer patients and their support network. Support Care Cancer. 2010;18(1):29-36.

3. Leydon GM, Guerin M, Eyles C, Jacques L, Darlison L. 'What matters to me': an international online survey of people treating, affected by and living with lung cancer.
Future Oncol. 2012;8(7):883-96.

4. Deveugele M. Communication training: Skills and beyond. Patient Educ Couns. 2015;98(10):1287-91.

5. Frankel RM, Sherman HB. The secret of the care of the patient is in knowing and applying the evidence about effective clinical communication. Oral Dis. 2015;21(8):91926.

6. Hitawala A, Flores M, Alomari M, Kumar S, Padbidri V, Muthukuru S, et al. Improving Physician-patient and Physician-nurse Communication and Overall Satisfaction Rates: A Quality Improvement Project. Cureus. 2020;12(4):e7776.

7. Brooks AT, Silverman L, Wallen GR. Shared decision making: a fundamental tenet in a conceptual framework of integrative healthcare delivery. Integr Med Insights. 2013;8:29-36.

8. Henselmans I, van Laarhoven HWM, van Maarschalkerweerd P, de Haes HCJM, Dijkgraaf MGW, Sommeijer DW, et al. Effect of a Skills Training for Oncologists and a Patient Communication Aid on Shared Decision Making About Palliative Systemic Treatment: A Randomized Clinical Trial. Oncologist. 2020;25(3):e57888.

9. Odai-Afotey A, Kliss A, Hafler J, Sanft T. Defining the patient experience in medical oncology. Support Care Cancer. 2020;28(4):1649-58.

10. Stiefel F, Barth J, Bensing J, Fallowfield L, Jost L, Razavi D, et al. Communication skills training in oncology: a position paper based on a consensus meeting among European experts in 2009. Ann Oncol. 2010;21(2):204-7.

11. Gilligan T, Bohlke K, Baile WF. PatientClinician Communication: American Society of Clinical Oncology Consensus Guideline Summary. J Oncol Pract. 2018;14(1):42-6.

12. Sobczak K, Leoniuk K, Janaszczyk A. Delivering bad news: patient's perspective and opinions. Patient Prefer Adherence. 2018;12:2397-404.

13. Seifart C, Hofmann M, Bär T, Riera Knorrenschild J, Seifart U, Rief W. Breaking bad newswhat patients want and what they get: evaluating the SPIKES protocol in Germany. Ann Oncol. 2014;25(3):70711.

14. Covvey JR, Kamal KM, Gorse EE, Mehta Z, Dhumal T, Heidari E, et al. Barriers and facilitators to shared decision-making in oncology: a systematic review of the literature. Support Care Cancer. 2019;27(5):1613-37. 
15. Mokhles S, Nuyttens JJME, de Mol M, Aerts JGJV, Maat APWM, Birim Ö, et al. Treatment selection of early stage non-small cell lung cancer: the role of the patient in clinical decision making. BMC Cancer. 2018;18(1):79.

16. Sullivan DR, Eden KB, Dieckmann NF, Golden SE, Vranas KC, Nugent SM, et al. Understanding patients' values and preferences regarding early stage lung cancer treatment decision making. Lung Cancer. 2019;131:47-57.

17. Banerjee SC, Manna R, Coyle N, Penn S, Gallegos TE, Zaider T, et al. The implementation and evaluation of a communication skills training program for oncology nurses. Transl Behav Med. 2017;7(3):61523.

18. Weeks JC, Catalano PJ, Cronin A, Finkelman MD, Mack JW, Keating NL, et al. Patients' expectations about effects of chemotherapy for advanced cancer. $\mathrm{N}$ Engl J Med. 2012;367(17):1616-25.

19. van der Gaag M, Heijmans M, Spoiala C, Rademakers J. The importance of health literacy for selfmanagement: A scoping review of reviews. Chronic Illn. 2021:17423953211035472.

20. Haase KR, Hall S, Sattar S, Ahmed S. Living with cancer and multimorbidity: A qualitative study of self-management experiences of older adults with cancer. Eur J Oncol Nurs. 2021;53:101982.

21. Berry LL, Danaher TS, Chapman RA, Awdish RLA. Role of Kindness in Cancer Care. J Oncol Pract. 2017;13(11):744-50.
22. Dawson RM, Lawrence K, Gibbs S, Davis V, Mele C, Murillo C. "I FELT THE CONNECTION": A QUALITATIVE EXPLORATION OF STANDARDIZED PATIENTS' EXPERIENCES IN A DELIVERING BAD NEWS SCENARIO. Clin Simul Nurs. 2021;55:52-8.

23. Fujimori M, Shirai $Y$, Asai M, Kubota K, Katsumata N, Uchitomi Y. Effect of communication skills training program for oncologists based on patient preferences for communication when receiving bad news: a randomized controlled trial. J Clin Oncol. 2014;32(20):2166-72.

24. Datta SS, Tripathi L, Varghese R, Logan J, Gessler S, Chatterjee S, et al. Pivotal role of families in doctor-patient communication in oncology: a qualitative study of patients, their relatives and cancer clinicians. Eur J Cancer Care (Engl). 2017;26(5).

25. Banerjee SC, Haque N, Bylund CL, Shen MJ, Rigney M, Hamann HA, et al. Responding empathically to patients: a communication skills training module to reduce lung cancer stigma. Transl Behav Med. 2021;11(2):613-8. 